\title{
Plasmonic nanopore-based platforms for single-molecule Raman scattering
}

\author{
Liang Deng ${ }^{\mathrm{a}, \mathrm{b}}$, Yixin Wang ${ }^{\mathrm{c}}$, Chen Liu ${ }^{\mathrm{a}, \mathrm{d}}$, Dora Juan Juan $\mathrm{Hu}^{\mathrm{c}}$, Perry Ping Shum ${ }^{\mathrm{e}}$ and Lei Su ${ }^{\mathrm{b}, \mathrm{a}, "}$ \\ ${ }^{a}$ Department of Electrical Engineering and Electronics, University of Liverpool, Liverpool L69 3GJ, United Kingdom \\ ${ }^{b}$ School of Engineering and Materials Science, Queen Mary University of London, London E1 4NS, United Kingdom \\ ${ }^{c}$ Institute for Infocomm Research, Agency for Science, Technology and Research (A*STAR), Singapore 138632, Singapore \\ ${ }^{d}$ School of Optical and Electronics Information, Huazhong University of Science and Technology, Wuhan 430074, China \\ ${ }^{e}$ School of Electrical \& Electronics Engineering, Nanyang Technological University, Singapore 639798, Singapore \\ Corresponding author: l.su@qmul.ac.uk
}

\begin{abstract}
We propose and demonstrate a novel plasmonic nanopore platform based on a bowtie-nanopore structure, for single-molecule sensing. In this nano structure, nano-bowties are integrated with solid-state nanopores to provide localized surface plasmon resonances for signal enhancement. We design and optimize the nano-structure by tuning both the bowtie gap and the bowtie angle, and investigate their influences on field enhancement, thereby achieving single-molecule sensitivity. In addition, we study the field enhancement by introducing an engineered photonic nano-cavity. This further strengthens the electric enhancement. An overall Raman enhancement factor of $2 \times 10^{8}$ is achieved in our simulation. This is believed to be sufficient for single-molecule sensing. The proposed bowtie-nanopore structure can be multiplexed on a single substrate for simultaneous multi-channel detection, paving the way for demanding applications such as DNA sequencing.
\end{abstract}

Keywords: nanopore; plasmonics; single-molecule Raman scattering

\section{Introduction}

Nanopore platforms are being extensively utilized in biochemical sensing for targets including nucleic acid [1], protein [2], virus [3] and heavy metal ions [4]. Compared with biological nanopores, solid-state nanopores are more chemically stable, flexible in size and compatible with today's semiconductor fabrication techniques, such as focused ion beam milling (FIB) [5], transmission electron microscope (TEM) [1] and electron-beam lithography (EBL) [6]. Furthermore, plasmonic structures (e.g. nanoantennas) can be patterned near the nanopores with techniques such as FIB and EBL to realize the so-called hot spots (i.e., areas with greatly enhanced electric field), which can be used to perform single-molecule sensing with surface enhanced Raman scattering (SERS) [7] or surface enhanced fluorescence (SEF) [8].

Real-time label-free sensing is highly desirable for biochemical and environmental applications. SERS offers rich structural information and is undoubtedly one of the most promising label-free detection techniques. To date, few works have been done concerning fluidic plasmonic nanopore sensing, although nanopore-based SERS substrates were demonstrated $[9,10]$. Also, in Ref $[11,12]$, metal deposited nanoholes were reported for the detection of certain analytes in fluidic environment. It is widely accepted that the electric enhancement due to surface plasmon resonance leads to a Raman enhancement factor of $10^{7}$, which is beyond the sensitivity of single molecule detections. Therefore, our study is motivated by the prospective of single-molecule sensing with nanopore, to introduce a nano-bowtie as a plasmonic optical nanoantenna around a nanopore to significantly enhance the detection limit. F. Nicoli et al. [13] investigated plasmonic nanopores with nanoantennas for DNA analysis but plasmonic excitation was to improve electric detection signals. To the best of our knowledge, the design in this work is the first fluidic nanoantenna-assisted nanopore platform. In addition, another exciting prospect of plasmonic nanopore systems is the potential of probing multiple nanopores simultaneously [14] for rapid and multi-channel detection, which is hard in nanopore-based electrical detection schemes [15].

In this work, we propose to use nanoantenna (in particular, nano bowtie) -assisted nanopore platforms for single-molecule SERS detection. Specifically, bowtie structures being used towards electromagnetic hotspots [16] are studied theoretically to achieve plasmonic electric field enhancement. The gap size as well as bowtie angle are studied for enhancement factor optimization. Additionally, the influence of the nanopore size on the nanoparticle-based plasmonic enhancement is investigated as the design guidance. Finally, photonic cavities are introduced into our system to further improve enhancement. Speaking of a reasonable instance, Raman enhancement factor of $2 \times 10^{8}$ is obtained with our optimized system. The proposed plasmonic nanopore platforms are then demonstrated capable of single-molecule SERS sensing.

\section{Theoretical Methods}


Bowtie-nanopore structures were designed and used in our plasmonic platforms, as shown in Fig. 1. The gold bowtie structure acting as a nanoantenna was placed on a silica substrate with its gap centre aligned with the nanopore centre. The nanopore works as a channel to allow the sample to pass and the localized surfaced plasmon resonance excited in the center of the nano-bowtie enhances Raman signals from the sample. At the same time, the silica substrate was used as spacer and a gold ground layer was added on the other side of the substrate to form a photonic cavity. In theory, a perfectly sharp tip at the apex of the triangle is most desirable. This, however, cannot be achieved by considering the limitations of EBL followed by vapor deposition of gold or similar fabrication techniques. In [17], effects of corner radius on field enhancement and resonance wavelength have been demonstrated. Therefore, we used a curvature (radius of $5 \mathrm{~nm}$ ) at the apex of the bowtie tip [18] in our simulation. A schematic drawing of the top view of the bowtie structure on $x y$-plane is shown in Fig. 1a while the cross section of the system on $x z$-plane is given in Fig. 1b. The dimension of the

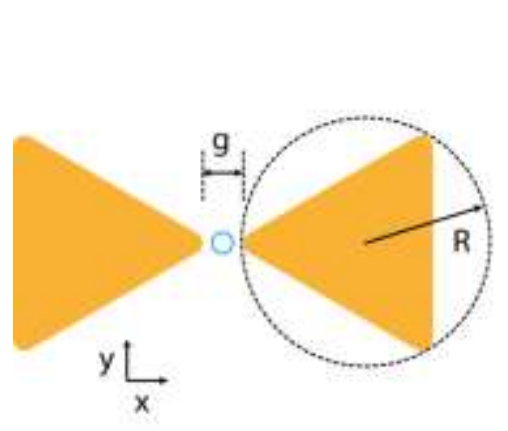

(a)
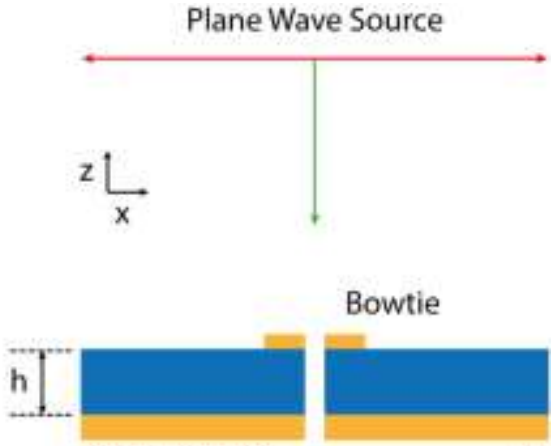

Gound Layer

\section{Bowtie}

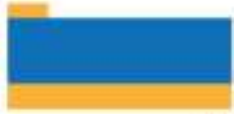

(b)

Fig. 1. Schematic drawing of (a) a bowtie with round corners on the $x y$-plane and (b) the side view of the system on the $x z$-plane.

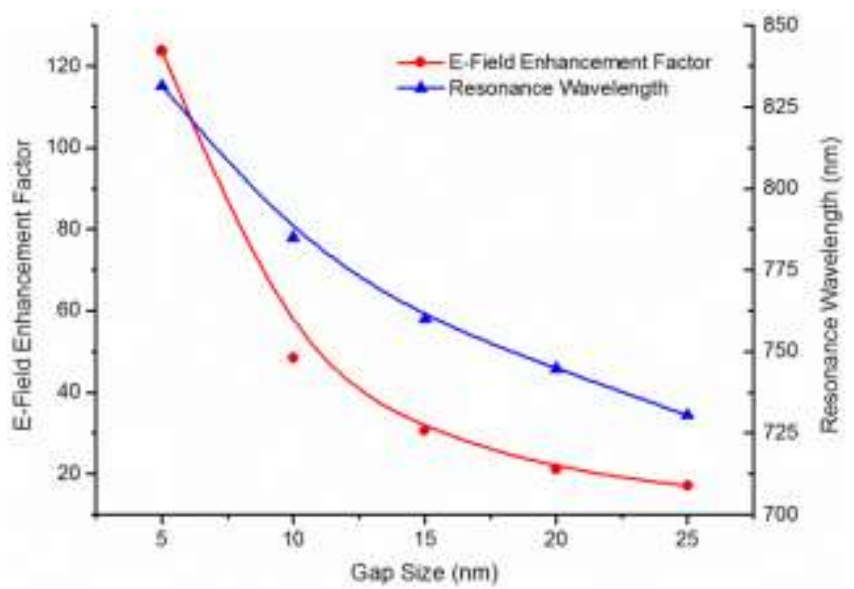

Fig. 2. Electrical field enhancement as a function of the bowtie gap size $(R=41.2 \mathrm{~nm})$

bowties is defined by $R$ (the radius of the circumcircle of the triangle prism). And $\phi$ stands for bowtie angle. In addition, $g$ and $h$ are the bowtie gap size and the silica substrate thickness, respectively. Thickness of the nanoantennas is $20 \mathrm{~nm}$ and that of the ground metal layer is $100 \mathrm{~nm}$. The working environment including the nanopore is filled with water.

Lumerical FDTD Solutions, the commercial electromagnetic software based on the finite-difference timedomain method, was used to perform the simulations. A plane wave polarized along the junction between triangular prisms (i.e., along the $x$ direction in Figure 1) was illuminated in the negative $z$-direction from above the bowtie (see Figure 1b). The perfect matched layers were used to absorb waves leaving the simulation domain in all wave propagation directions. The dielectric property of Au used in the simulations was taken from the John and Christy's report [19] and the $\mathrm{SiO}_{2}$ information was achieved from Palik's handbook [20]. The mesh size in the bowtie region (including the gap) was $1 \mathrm{~nm}$ while automatic graded mesh was adopted in the region outside the bowtie structures to ensure the numerical accuracy in consideration of reasonable computation time.

\section{Results and Discussions}

We firstly studied the gap dependence of nanoantenna performance. Gold ground layer was not introduced into our system at this stage and its influence was investigated separately later. Fig. 2 shows the change of resonance wavelength as well as electric field enhancement factor of standard bowties (adapted from regular triangles) at the gap centre corresponding to different gap size but with same nanopore (diameter of $5 \mathrm{~nm}$ ). Clearly, by decreasing the size of the gap, the resonance wavelength shifts to longer wavelengths and a stronger electric 
field was obtained. The red-shift of resonance wavelength is owing to the decreasing of resonance frequency of dipoles getting closer as a result of charge attraction. The enhancement of electric field results from stronger plasmonic coupling with narrower bowtie feed gap. It is worth mentioning that sub-10 nm solid-state nanopores and feed gaps (gap size should not be smaller than pore diameter) are hard to obtain on substrates with today's fabrication techniques $[1,21,22]$. However, extremely narrow bowtie gap $(5 \mathrm{~nm}$ here, limited by techniques and pore diameter) does lead to giant electric field enhancement (120 times stronger as shown in Fig. 2).

In addition to the gap size, the impact of bowtie angle was also studied and the results are displayed in Fig. 3. The bowtie with an angle of 60 degree is the standard bowtie investigated in Fig. 1. Note that all the bowties here are with same surface area for accurate comparison. Obviously, with the increase of bowtie angle, the electric

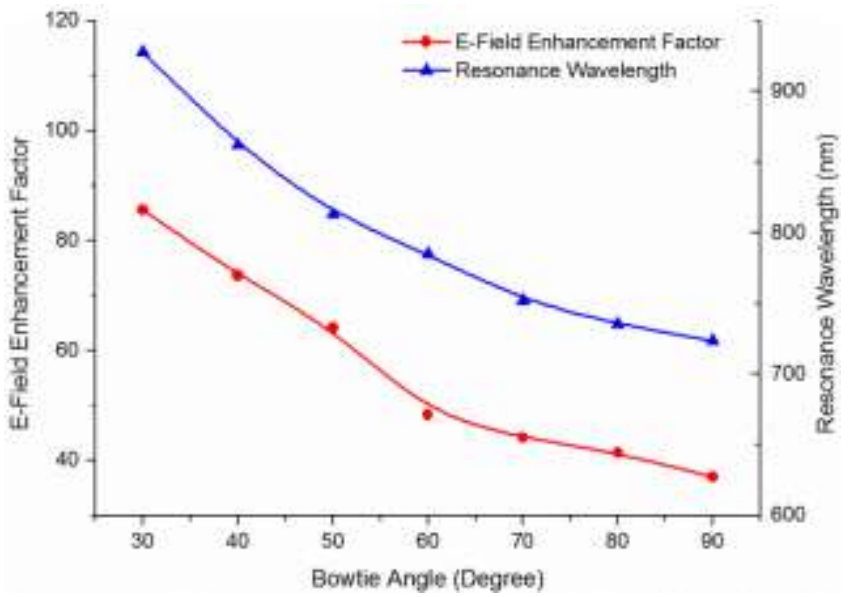

Fig. 3. Electrical field enhancement as a function of the bowtie angle

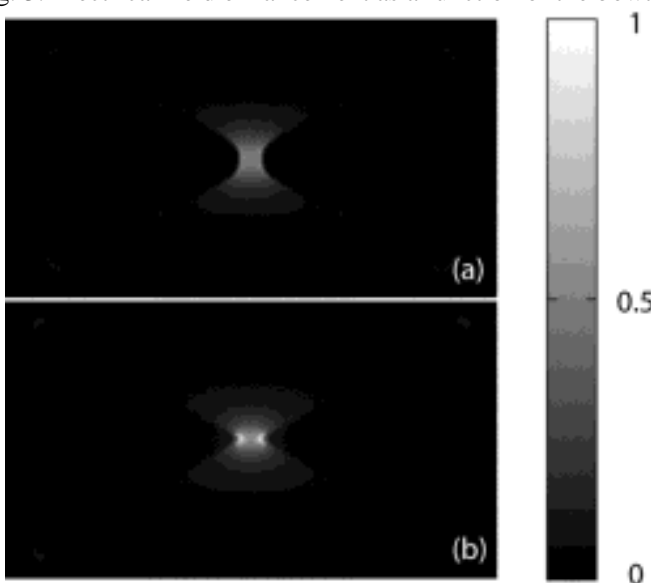

Fig. 4. Normalized electric field distributions of (a) a round bowtie and (b) an ideal bowtie

field enhancement decreases and the resonance wavelength blue-shifts. For instance, electric field enhancement could be doubled with 30-degree bowtie compared to a 60-degree bowtie. But it suffers from dramatic shifting of resonance wavelength (over $100 \mathrm{~nm}$ ). The hot spots in nano-plasmonics is due to the lightning rod effect generating high local field at a sharp tip when the polarization of the exciting light is parallel to the tip's axis. That is the reason why our plane wave is polarized along bowtie junction. Furthermore, the lightning rod effect becomes stronger with even sharper tips, which explains the change of enhancement factor in Fig. 3. Stronger plasmonic coupling accompanies with longer working wavelength as shown in Fig. 1. In addition, comparisons between round and ideal bowties of 5-nm gap are presented in Fig. 4. It is clear that ideal bowties could lead to even hotter spots around sharp tips, which is consistent with lightning-rod effect.

It is also worth investigating the influence of nanopore size as it is closely related to the size of the analyte. We compared $20 \mathrm{~nm}$-gap standard bowties with different size of nanopores and corresponding results are given in Fig. 5. It can be seen that the field enhancement and the resonance wavelength of the bowtie nanoantennas are relatively stable over a range of nanopore sizes. We attribute this to the fact that nanoantennas' features depend mainly on the bowties, such as their material, shape, size. Therefore, we can conclude that the introduction of nanopores has limited impact on the given nanoantenna. This provides a useful guideline in the design and applications of such plasmonic nanopore platforms.

Photonic cavities, formed by adding another metal layer under substrates, have been reported to further improve field enhancement [23]. Here we studied the enhancement produced by photonic cavities with the 10nm-gap standard bowtie used in Fig. 1. Here, a $785 \mathrm{~nm}$ excitation laser was chosen since such a wavelength is 
commonly used in Raman spectroscopy, and fits into the resonance wavelength of the chosen antenna. The change of electric field enhancement factors with different silica substrate thickness is shown in Fig. 6. According to Ref. [23], the strongest enhancement occurs at the quarter-wavelength optical thickness due to constructive interference. Because of $\pi$ phase shift during reflection at the interface of a Perfect Electric Conductor (PEC) and dielectric material, the in-phase reflection condition is expressed as follows:

$$
2 \times h \times n_{\text {silica }}=\left(m+\frac{1}{2}\right) \lambda
$$

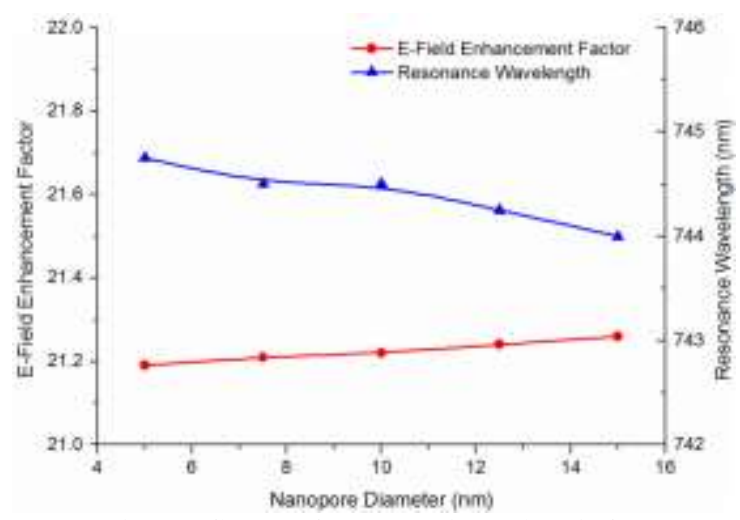

Fig. 5. Influence of nanopore size on bowtie features

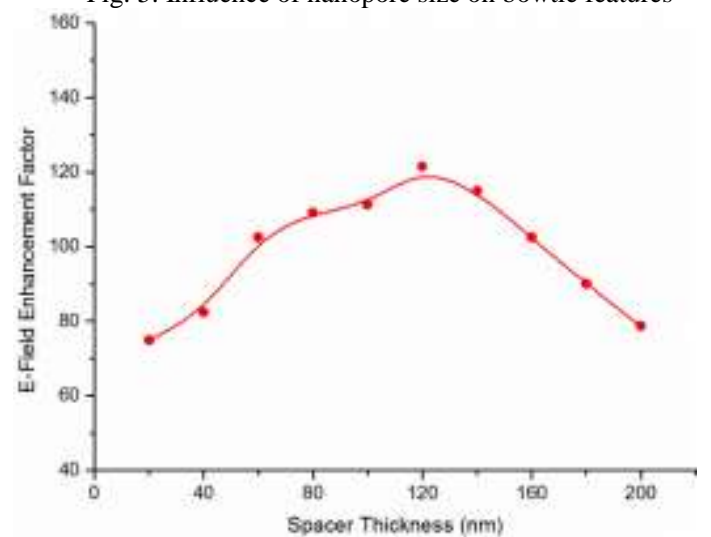

Fig. 6. Electrical field enhancement as a function of spacer thickness

where $n_{\text {silica }}$ is the index of silica substrate; $\lambda$ denotes laser wavelength and $m$ is a whole number. By considering the parameter of our system (laser wavelength of $785 \mathrm{~nm}$, silica index of 1.45), the first order of optimized thickness is around $135 \mathrm{~nm}$. It is found in Fig. 6 that the actual optimized thickness $(120 \mathrm{~nm})$ is slightly less than that calculated value. This is because gold is not perfect electric conductor and the penetration of laser light into the gold layer leads to additional phase shift at the metal ground layer-silica substrate interface. In our simulation, the largest electric field enhancement observed is over 120, providing an additional electric-fieldenhancement factor of 2.5, compared to the original enhancement factor of 48 in Fig. 2. If both the Stokes shift and the loss are negligible, the Raman enhancement factor can be approximated as:

$$
E F_{\text {Raman }} \approx\left|\frac{E_{\text {loc }}\left(f_{0}\right)}{E_{\text {in }}\left(f_{0}\right)}\right|^{4}
$$

where $E_{\text {in }}\left(f_{0}\right)$ is the incident electric field at laser frequency, $E_{\text {loc }}\left(f_{0}\right)$ denotes the local electric field at laser frequency This approximation was proved in [24] and was therefore used to evaluate our system here. Therefore, additional Raman enhancement factor is $2.5^{4} \approx 40$ and total Raman enhancement factor is over $2 \times 10^{8}$ which evidently satisfies the requirement for single-molecule SERS $\left(10^{7} \sim 10^{8}\right)$ [25]. It is worth mentioning that this figure was obtained based on considerations of limitations on available fabrication technologies. The enhancement will be much larger with narrower bowtie gap and smaller bowtie angle. Additionally, excitation wavelength is another important consideration and will be determined by the size of nanoantenna.

Our structure can be developed into a multi-channel device if periodic bowties and nanopores are fabricated on the same substrate. For periodic structures, the resonance wavelength will experience red shift [26], which means further design considerations are needed to keep the bowtie working at $785 \mathrm{~nm}$. Besides, nano bowtie arrays could offer larger enhancement by selecting an appropriate period. It is supposed to be the laser wavelength, as is explained by the so-called long-range collective photonic effect. It was theoretically predicted in [27] and demonstrated experimentally with bowtie arrays in [28]. By using water as the working environment, 
the optimized period is $785 \mathrm{~nm} / 1.33 \approx 590 \mathrm{~nm}$. The proposed fabrication process is presented in Fig. 7, where nano-fabrication techniques, such as etching, EBL and FIB, can be used to produce the designed structure. The thick silicon substrate is etched to leave a thin silica layer with both sides sputtered with a gold layer (one side is for bowtie and the other acts as a ground layer). EBL can be used to produce the nanobowtie and FIB is proposed to generate the aligned nanopore.

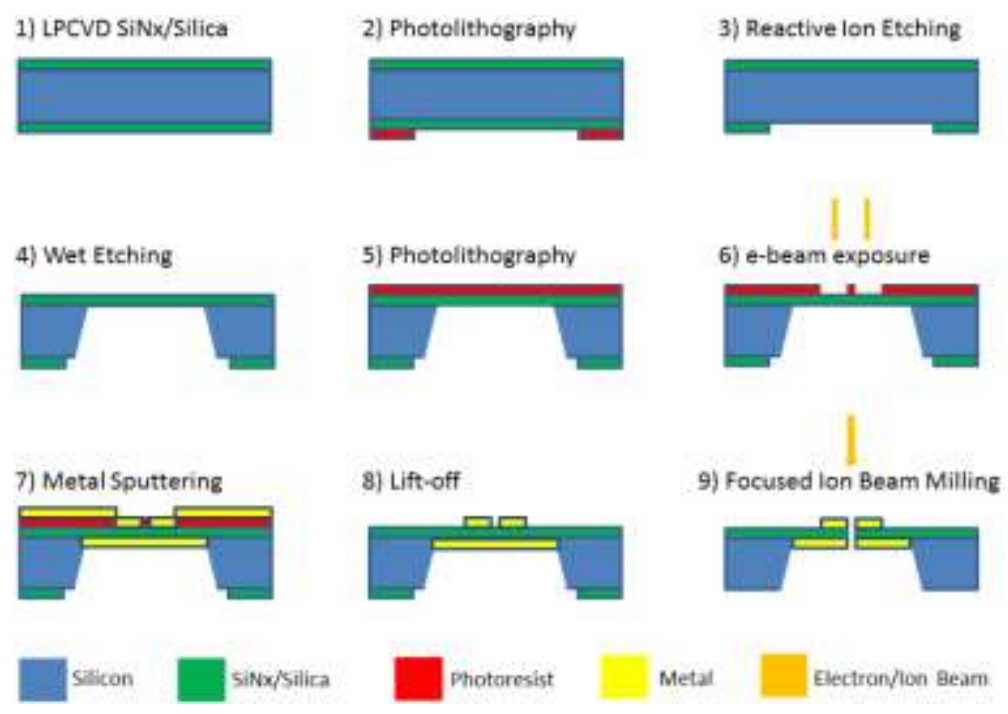

Fig. 7 Proposed fabrication process

\section{Conclusion}

In summary, we proposed and investigated theoretically a novel plasmonic nanopore platform, consisting of nano-antennas and a nanopore, suitable for single-molecule SERS sensing. The influence of bowtie properties and nanopore size were studied in order to guide the design and optimize the electrical field enhancement. We found a narrower gap and a sharper bowtie angle lead to stronger plasmonic electric enhancement. Also, a metal ground layer was introduced into the system to form a photonic cavity to further improve the enhancement factor. A platform example was evaluated, and a Raman enhancement factor of $2 \times 10^{8}$ was obtained, demonstrating the ability for single-molecule SERS detection. The proposed plasmonic nanopore platform can be designed for specific analytes, can be multiplex for multichannel simultaneous sensing, holding the promise in future fluidic single-molecule SERS applications.

\section{References}

[1] M. Wanunu, T. Dadosh, V. Ray, et al., Nat Nanotechnol 5 (2010) 807.

[2] W. Li, N.A. Bell, S. Hernández-Ainsa, et al., ACS NANO 7 (2013) 4129.

[3] A. McMullen, H.W. de Haan, J.X. Tang, et al., Nat Commun 5 (2014) 4171.

[4] C.-H. ZHANG, G.-J. LI, J.-H. WANG, Chin J Anal Chem 42 (2014) 607.

[5] C.A. Volkert, A.M. Minor, MRS Bull 32 (2007) 389.

[6] V.R. Manfrinato, L. Zhang, D. Su, et al., Nano Lett 13 (2013) 1555.

[7] S.S. Acimovic, M.P. Kreuzer, M.U. González, et al., Nano Lett 3 (2009) 1231.

[8] P. Anger, P. Bharadwaj, L. Novotny, Phys Rev Lett 96 (2006) 113002.

[9] D. Choi, Y. Choi, S. Hong, et al., Small 6 (2010) 1741.

[10] P.D. Robinson, A. Kassu, A. Sharma, et al., J Photonics 7 (2013) 073592.

[11] C. Chen, J. Ye, Y. Li, et al., IEEE J Sel Top Quant Electron 19 (2013) 4600707.

[12] S. Kumar, S. Cherukulappurath, T.W. Johnson, et al., Chem Mater 26 (2014) 6523.

[13] F. Nicoli, D. Verschueren, M. Klein, et al., Nano Lett 14 (2014) 6917.

[14] B. McNally, A. Singer, Z. Yu, et al., Nano Lett 10 (2010) 2237.

[15] T. Osaki, H. Suzuki, B. Le Pioufle, et al., Anal Chem 81 (2009) 9866

[16] S. Dodson, M. Haggui, R. Bachelot, et al., Phys Chem Lett 4 (2013) 496.

[17] B.K. Chao, S.C. Lin, L.W. Nien, et al., J Opt 17 (2015) 125002.

[18] L.W. Nien, S.C. Lin, B.K. Chao, et al., J Phys Chem C 117 (2013) 25004.

[19] P.B. Johnson, R.W. Christy, Phys Rev B: Solid State 6 (1972) 4370.

[20] E.D. Palik, Handbook of Optical Constants of Solids III, Academic Press, San Diego, 1998.

[21] W. Zhu, M.G. Banaee, D. Wang, et al., Small 7 (2011) 1761.

[22] H. Kollmann, X. Piao, M. Esmann, et al., Nano Lett 14 (2014) 4778.

[23] Q. Min, Y. Pang, D.J. Collins, et al., Opt Express 19 (2011) 1648.

[24] E.C. Le Ru, P.G. Etchegoin, Chem Phys Lett 423 (2006) 63.

[25] H.M. Lee, S.M. Jin, H.M. Kim, et al., Phys Chem Chem Phys 15 (2013) 5276.

[26] K. Leong, M.T. Zin, H. Ma, et al., ACS Appl Mater Inter 2 (2010) 3153.

[27] K. Zhao, H. Xu, B. Gu, et al., J Chem Phys 125 (2006) 081102.

[28] N.A. Hatab, C. Hsueh, A.L. Gaddis, et al., Nano Lett 10 (2010) 4952. 